\title{
Deep Learning-Based Pest Surveillance System for Sericulture
}

\author{
Pramodh BR \\ Data Scientist, \\ Bangalore, India \\ Email:pramodhbr29@gmail.com
}

\author{
Thippesha D iD \\ IEEE Member \\ Bangalore section, India. \\ Email: thippesh790@gmail.com
}

\begin{abstract}
:
Every year sericulture farmers lose a sizeable amount of revenue because of pest attacks on silkworms. In 2011 the annual production of silk is fall by about $50 \%$ because of pest attacks [1]. To prevent these losses constant monitoring of the environment is required. But this constant surveillance can't be achievable by manual labour force but it can be achievable by using deep learning techniques. This article presents a deep learning system that is trained and tested for detecting invasive species which can cause harm to silkworms such as Oecophylla smargdina, Vespa orientalis, Sycanus collaris, Hierodulla bipapilla, Canthecona furcellata, Blepharipa zebina and Apanteles glomeratus.
\end{abstract}

\section{Keywords:}

Silkworm, Deep learning, Inception ResNet, Inception-V3, VGG16, VGG19, Oecophylla smargdina, Vespa orientalis, Sycanus collaris, Hierodulla bipapilla, Canthecona furcellata, Blepharipa zebina, Apanteles glomeratus.

\section{Introduction:}

Clothing is one of the basic needs of the man it not only protects the man from coldness is also a symbol of pride and status. Silk is one such example of it. In basic, the silk is obtained from the cocoon of the silkworm. The quality of the silk cocoons decides its cost; any abnormalities in the silk cocoons will affect the price. Hence to obtain that highest quality it's necessary to control every parameter its starts from feeding good quality food to securing the cocoons from diseases and pests [2] as in Table 1 .

The finest quality food can be made available by growing the best mulberry pants in the right condition. The various diseases can be prevented using antibiotics and genetically modifying silkworms. But preventing the pests is a challenging and complex process because preventing the pests cannot be done by just using the chemicals or pesticides and insecticides because these chemicals also affect the health of the silkworm. And using the same chemicals again and again those pests may develop a resistive power for that particular chemical [3].

The effective way to prevent these types of pests is to use the preventive measure as early as possible. But the pests are prevented before infecting the silkworm in any manner is very difficult because of the nature of these insects as in fig 1. Most of the intruding species are having capabilities such as small size, flying and camouflage some possess all these characters.

Hence it needs a continuous monitoring system to alert about these kinds of pest intrusions this can be done by using the existing hardware devices but the logical requirements can only be filled by artificial intelligence because of the complexity of the process.

Table 1. Various pests of silkworms

\begin{tabular}{|c|c|c|}
\hline Category & $\begin{array}{l}\text { Scientific } \\
\text { Name }\end{array}$ & Invasion Type \\
\hline Ants & $\begin{array}{l}\text { Oecophylla } \\
\text { smargdina }\end{array}$ & $\begin{array}{l}\text { Attack and kill numbers of early } \\
\text { larval instars of tropical Tasar } \\
\text { silkworm }\end{array}$ \\
\hline \multirow[t]{2}{*}{ Parasites } & $\begin{array}{l}\text { Apanteles } \\
\text { glomeratus }\end{array}$ & $\begin{array}{l}\text { Infects the early stage } \\
\text { silkworms }\end{array}$ \\
\hline & $\begin{array}{l}\text { Blepharipa } \\
\text { zebina }\end{array}$ & $\begin{array}{l}\text { Lives inside silkworm in the } \\
\text { larval stage and ultimately kills } \\
\text { it }\end{array}$ \\
\hline \multirow[t]{3}{*}{ Predators } & $\begin{array}{l}\text { Canthecona } \\
\text { furcellata }\end{array}$ & $\begin{array}{l}\text { Feed for longer duration on } \\
\text { Tasar silkworm }\end{array}$ \\
\hline & $\begin{array}{l}\text { Hierodulla } \\
\text { bipapilla }\end{array}$ & Feeding on Tasar silkworm \\
\hline & $\begin{array}{l}\text { Sycanus } \\
\text { collaris }\end{array}$ & $\begin{array}{l}\text { Attack to silkworms in the } \\
\text { rearing field }\end{array}$ \\
\hline Wasps & $\begin{array}{l}\text { Vespa } \\
\text { orientalis }\end{array}$ & Attack to silkworms to eat \\
\hline
\end{tabular}




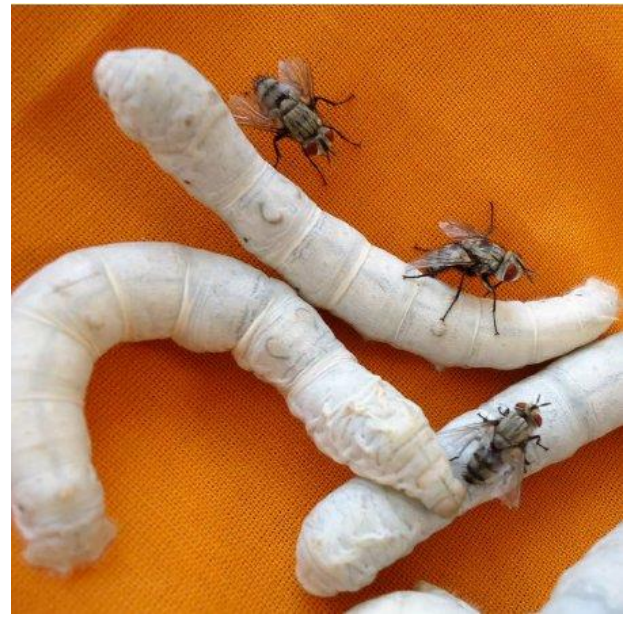

Fig 1. Uzi fly infesting the silkworm [4]

\section{Literature survey:}

In [5] the Reddy, Bontha Kasi, and Jemmy Venkata Krishna Rao conducted A three-year survey of 150 sericulture farmers in the districts of Chittoor and Anantapur in Andhra Pradesh on the occurrence of silkworm illnesses such as Grasserie, Flacherie, Muscardine, and Uzi fly. The survey concluded that proper care and precautions can prevent pest attacks effectively. Vijay Veer, B.K. Negi, K.M. Rao, conducted research [6] on pests that can cause serious damages to the larva and silkworms. And also identified new species of pests which were not documented before such as Tribolium castaneum.

X. Wan, F. Ren and D. Yong [7] used an Inception ResNet based system to estimate the age of the person with help of image data. The proposed system is intended to use preventing bank fraud and fake ID cards. Xiaoling Xia, Cui Xu and Bing Nan [8] trained a flower classification system based on the inception $v 3$ algorithm which can differentiate between identical species without any bias. The system can be used in botany related fields for identifying plant species. T. Kaur and T. K. Gandhi developed a brain image classification system [9] that can be used to diagnose neurological disorders by analysing MRI images. This system is trained using the VGG16 algorithm. M. Shaha and M. Pawar had done a survey [10] on transfer learning for image classification using VGG19 AlexNet and VGG16. The models are trained with ImageNet data sets and their performance has been recorded and compared with other algorithms performance.

\section{Methodology:}

A) Data acquisition: The image files were collected from the internet and manually sorted and numbered manually with help of a data annotator. These files are stored in a local server which can be accessed by the machine. The data is split into train data and test data with a ratio of 7:3.

B) Data augmentations: Data augmentation was used to enhance the learning process. This was done using an open-source program GIMP and with an added plugin of "Bimp for GIMP". And these files are added to the data repository.

C) Training the model: The model is trained using python 3 with Inception ResNet, Inception V3, VGG 16 and VGG 19 algorithms. All four algorithms are fed with the same data. Time is taken to train, Loss, Accuracy, Validation Loss and Validation Accuracy were noted down for the analysis.

\section{Results:}

The best algorithm is decided by the comparison of the key parameters such as the time taken to train as in fig 2, accuracy as in fig 3, validation accuracy as in fig 4 , loss as in fig 5 and validation loss as in fig 6. It appears that all the four algorithms did cross the 95\% accuracy, but the Inception V3 algorithm performs best it consumes a very short time for training and also has the best accuracy score and validation accuracy score. And it reached its benchmark in less time compared to other algorithms. The Inception ResNet algorithm took 15 epochs to reach above $95 \%$ accuracy the validation loss is also high compared to other algorithms. The VGG16 and VGG19 consumed more time for each epoch compared to Inception V3 and Inception ResNet.

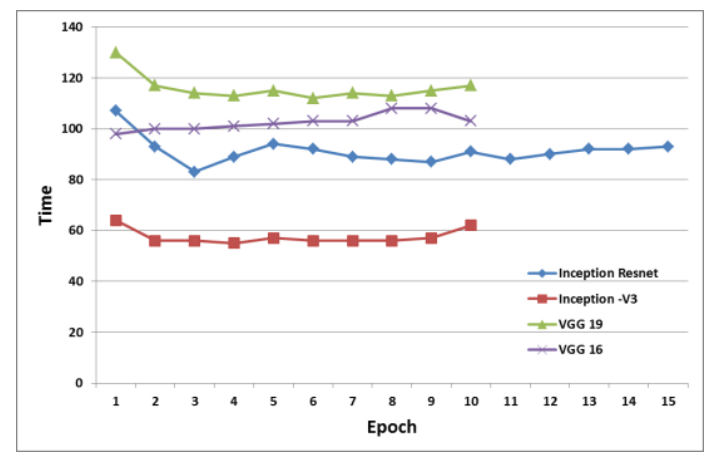

Fig 2. Time is taken by the algorithms 


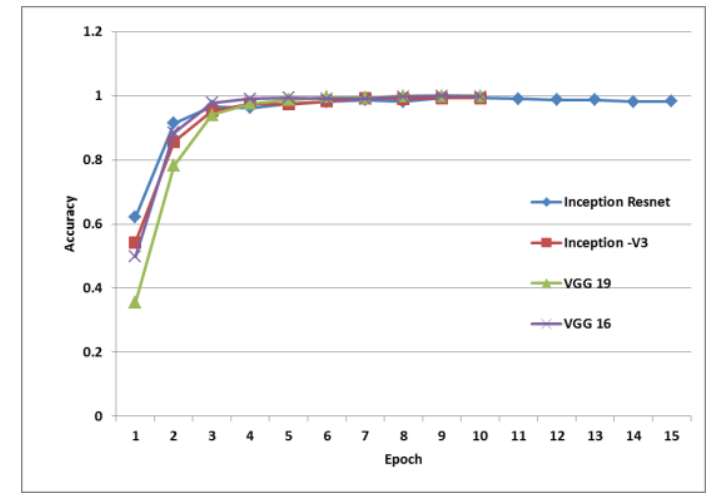

Fig 3. Accuracy of algorithms

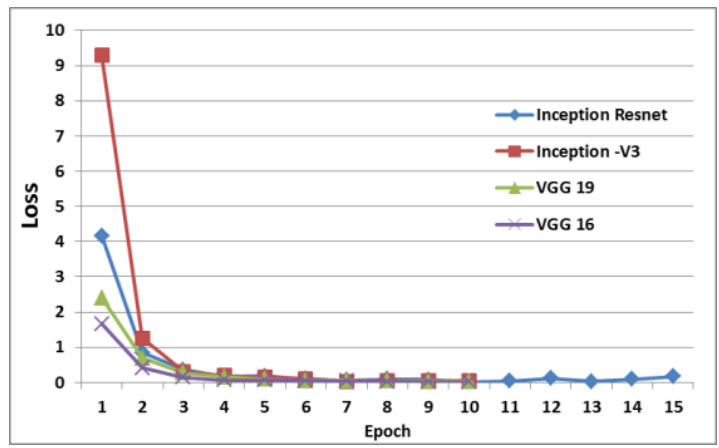

Fig 5. Loss of algorithms

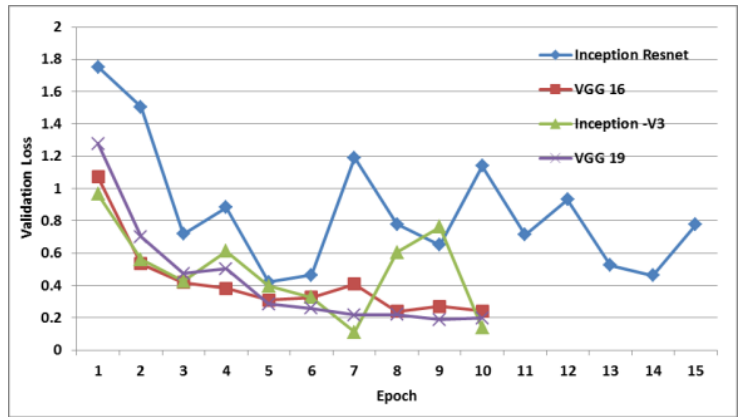

Fig 6. Validation Loss of algorithms

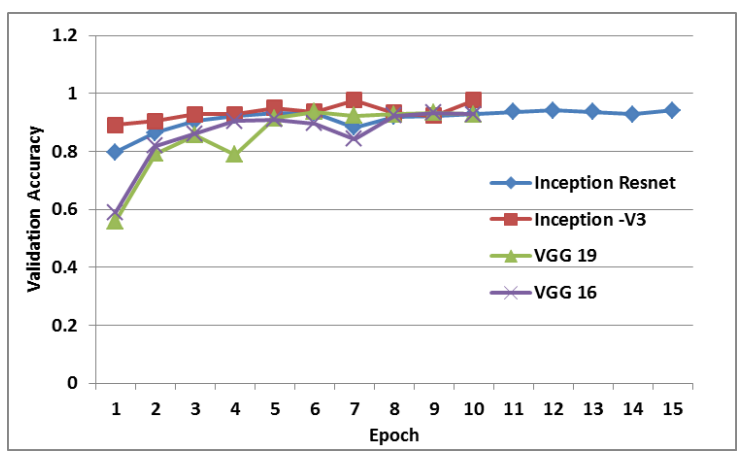

Fig 4. Validation Accuracy of algorithms

The AI model trained using Inception V3 was deployed and tested for feasibility and it performed with an appreciable confidence level in detecting pests as in fig 7 .

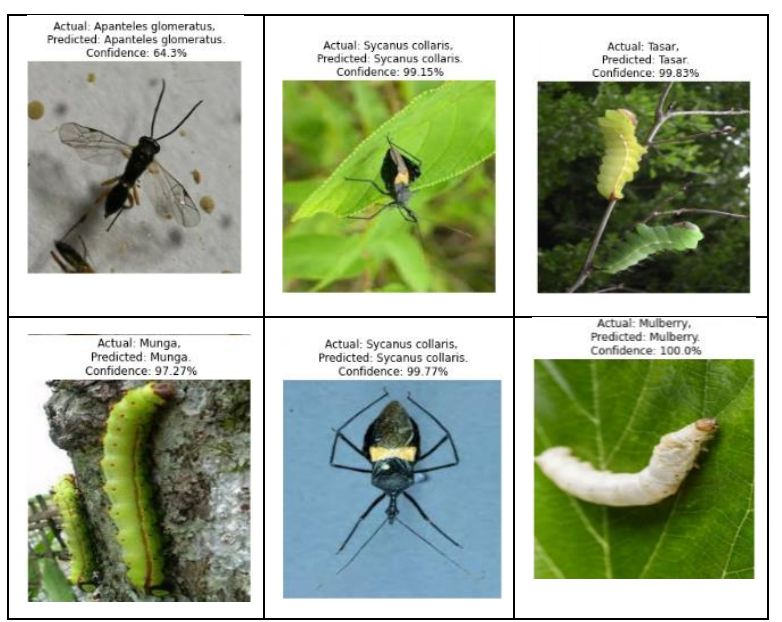

Fig 7. Deployed system classifying pest and silkworms

\section{Conclusion:}

The pests attack on silkworms can be prevented by using a deep learning-based surveillance system that detects the presence of the past with help of a camera and alerts the farmer to take necessary precautions in the early stage of their attack. In the future, a network of pest surveillance systems can help to rescue other crops from pests such as Locusts by predicting their moment from one location to another location in real-time.

\section{References:}

[1]Financialexpress.com

https://www.financialexpress.com/archive/silk-output-likely-tofall-due-to-pest-attacks/792742/

[2] Andiappan, Selvaraj \& Vijaysanthi, K. (2011). A Study on the Price Behavior of Cocoon and Raw Silk in Tamil Nadu. The IUP Journal of Agricultural Economics. VIII. 28-40.

[3] Yu, S.J. 2008. The Toxicology and Biochemistry of Insecticides. CRC Press, Boca Raton

[4] M., Prakash, \& Prathima, Aswathaiah \& Ravikumar, Hemagirigowda \& Hoolageri, Huchesh \& Hp, Puttaraju. (2013). Metapopulation biology of the Indian uzi fly, Exorista sorbillans (Wiedemann) (Diptera: Tachinidae). The Tachinid Times. 4649.

[5] Reddy, Bontha Kasi, and Jemmy Venkata Krishna Rao. "Seasonal occurrence and control of silkworm diseases, grasserie, flacherie and muscardine and insect pest, uzi fly in Andhra Pradesh, India." International Journal of Industrial Entomology 18, no. 2 (2009): 57-61. 
[6] Vijay Veer, B.K. Negi, K.M. Rao, "Dermestid beetles and some other insect pests associated with stored silkworm cocoons in India, including a world list of dermestid species found attacking this commodity", in Journal of Stored Products Research, Volume 32, Issue 1, 1996, Pages 69-89, ISSN 0022474X, https://doi.org/10.1016/0022-474X(95)00032-3.

[7] X. Wan, F. Ren and D. Yong, "Using Inception-Resnet V2 for Face-based Age Recognition in Scenic Spots," 2019 IEEE 6th International Conference on Cloud Computing and Intelligence Systems (CCIS), 2019, pp. 159-163, doi: 10.1109/CCIS48116.2019.9073696.

[8] Xiaoling Xia, Cui Xu and Bing Nan, "Inception-v3 for flower classification," 2017 2nd International Conference on Image, Vision and Computing (ICIVC), 2017, pp. 783-787, doi: 10.1109/ICIVC.2017.7984661

[9] T. Kaur and T. K. Gandhi, "Automated Brain Image Classification Based on VGG-16 and Transfer Learning," 2019 International Conference on Information Technology (ICIT), 2019, pp. 94-98, doi: 10.1109/ICIT48102.2019.00023.

[10] M. Shaha and M. Pawar, "Transfer Learning for Image Classification," 2018 Second International Conference on Electronics, Communication and Aerospace Technology (ICECA), 2018, pp. 656-660, doi: 10.1109/ICECA.2018.8474802 